\title{
Dynamical evolution of anisotropies of the solar wind magnetic turbulent outer scale
}

\author{
M. E. Ruiz ${ }^{1}$, S. Dasso ${ }^{1,2}$, \\ W. H. Matthaeus ${ }^{3}$, E. Marsch ${ }^{4}$ and J. M. Weygand ${ }^{5}$ \\ ${ }^{1}$ Instituto de Astronomía y Física del Espacio (CONICET-Universidad de Buenos Aires), C.C. \\ 67, Sucursal 28, 1428, Buenos Aires, Argentina. email: meruiz@iafe.uba.ar \\ ${ }^{2}$ Departamento de Física, Facultad de Ciencias Exactas y Naturales, Universidad de Buenos \\ Aires, Pabellón 1 (1428), Buenos Aires, Argentina. email: dasso@df .uba.ar \\ ${ }^{3}$ Bartol Research Institute, Department of Physics and Astronomy, University of Delaware, \\ Newark, DE, USA. email: whm@udel.edu \\ ${ }^{4}$ Max-Planck-Institut für Sonnensystemforschung, Max-Planck-Straße 2, Katlenburg-Lindau, \\ Germany. email: marsch@mps.mpg.de \\ ${ }^{5}$ Institute of Geophysics and Planetary Physics, University of California, Los Angeles, CA, \\ USA. email: jweygand@igpp.ucla.edu
}

\begin{abstract}
The evolution of the turbulent properties in the solar wind, during the travel of the parcels of fluid from the Sun to the outer heliosphere still has several unanswered questions. In this work, we will present results of an study on the dynamical evolution of turbulent magnetic fluctuations in the inner heliosphere. We focused on the anisotropy of the turbulence integral scale, measured parallel and perpendicular to the direction of the local mean magnetic field, and study its evolution according to the aging of the plasma parcels observed at different heliodistances. As diagnostic tool we employed single-spacecraft correlation functions computed with observations collected by Helios 1 \& 2 probes over nearly one solar cycle. Our results are consistent with driving modes with wave-vectors parallel to the direction of the local mean magnetic field near the Sun, and a progressive spectral transfer of energy to modes with perpendicular wave-vectors. Advances made in this direction, as those presented here, will contribute to our understanding of the magnetohydrodynamical turbulence and Alfvénic-wave activity for this system, and will provide a quantitative input for models of charged solar and galactic energetic particles propagation and diffusion throughout the inner heliosphere.
\end{abstract}

Keywords. Solar wind, turbulence, magnetohydrodynamics.

\section{Introduction}

The solar wind (SW) is a turbulent plasma and the fluctuations of their bulk properties are ussually studied within the framework of magnetohydrodynamic (MHD) turbulence.

As originally proposed by Belcher \& Davis (1971), it is often considered that the main source of the interplanetary fluctuations is near the Sun, below the alfvénic critical point. Near 0.3 astronomical unit (AU) from the Sun, fluctuations are observed to be highly alfvénic with the sign of the cross helicity indicating mainly outward propagation from the Sun. Nevertheless, Coleman's (1968) point of view of an evolving turbulent $\mathrm{SW}$ is essential for the evolution of the turbulence throughout the heliosphere. Velocity gradients at large scales can drive local nonlinear instabilities that inject kinetic energy only (i.e., injection of zero cross helicity turbulence, leading to a decrease of cross helicity with heliodistance and to a state of well developed turbulence (Roberts et al. 1992).

Solar wind turbulence is anisotropic with respect the mean magnetic field $\mathbf{B}_{0}$ as has been suggested in many studies (Robinson \& Rusbridge 1971; Shebalin et al. 1983; 
Oughton et al. 1974; Goldreich \& Sridhar 1995). The simplest models commonly used for the description of anisotropic SW fluctuations are the slab model, where fluctuations have wavevectors parallel to $\mathbf{B}_{0}$, and the $2 \mathrm{D}$ model where fluctuations have wavevectors perpendicular to $\mathbf{B}_{0}$. These models, although very simplified, provide a useful parametrization of anisotropy in SW turbulence, in the sense that all wavevector contributions can be grouped into these two categories.

The solar wind three-dimensional structure is highly dependent upon the solar cycle (see, e.g. McComas et al. (2003)). During low solar activity, the SW presents a bi-modal structure, with a regular fast wind at high latitudes and a much more variable slow wind at low latitudes. On the contrary, near solar maximum, the wind structure is more complex, being highly variable at all heliolatitudes and arising from very diverse sources. Thus, it is reasonably to expect a stronger velocity shear during solar maximum than during minimum.

Then, one would like to know if the solar cycle, through this shear mechanism, assuming identical turbulent initial conditions near the Sun, can affect the SW turbulence properties?

In order to explore this possible scenario, we look at anisotropies in the correlation scale of the turbulence, and examine its evolution with heliodistance for different periods of time, thereby taking into account the dynamical age of the turbulence as well as the magnetic field direction and SW speed. To address this question, we employ Helios 1 (H1) \& Helios 2 (H2) observations made over almost a complete 11-year solar cycle. These are unique spacecrafts since they have systematically explored the inner heliosphere providing us with the youngest samples of wind observed in situ.

In the following, we briefly present the theoretical background for this work, describe the data processing, analyze the anisotropy in the correlation scale, and present and discuss the results.

\section{Correlation lengths: analysis, results and discussion}

The magnetic autocorrelation function $R$ is commonly employed in studies of turbulent magnetofluids like the SW (e.g., Tu \& Marsch (1995)).

Assuming homogeneity in space and time, $R$ can be defined as:

$$
R(\mathbf{r}, \tau)=\langle\mathbf{b}(\mathbf{x}, t) \cdot \mathbf{b}(\mathbf{x}+\mathbf{r}, t+\tau)\rangle
$$

where $\mathbf{r}$ and $\tau$ are the spatial and temporal lags respectively.

Observations made by only one probe allows us to calculate $R$ in the $\mathrm{SW}$ as $R\left(-\mathbf{V}_{\mathrm{sw}} \tau, \tau\right)$, with $\mathbf{V}_{\text {sw }}$ the SW velocity.

However, due to the superalfvénic character of the SW, it is possible to construct spatial correlation functions by means of the frozen-in flow Taylor hypothesis: $R_{b b}\left(-\mathbf{V}_{\mathbf{s w}} \tau, \tau=\right.$ $0)=R_{b b}(\mathbf{r}=\mathbf{0}, \tau)$ (Taylor 1938). Then the intrinsic lag time dependence in equation 2.1 can be ignored and $R$ becomes a function of $\mathbf{r}$ alone, giving $R(\mathbf{r})=\langle\mathbf{b}(\mathbf{0}) \cdot \mathbf{b}(\mathbf{r})\rangle$.

As mentioned before, anisotropies will develop with respect to $\mathbf{B}_{0}$ and therefore correlation functions will not behave in the same way in different directions. A measure of this correlation anisotropy can be given by means of the integral scale $\lambda$ computed from $R$. If we consider a spatial lag $\mathbf{r}(\theta)$ making an angle $\theta$ with respect to $\mathbf{B}_{0}$, the correlation lengths in the $\hat{\mathbf{r}}(\theta)$ direction can be defined as:

$$
\lambda(\theta)=\frac{\int_{0}^{\infty}\langle\mathbf{b}(\mathbf{0}) \cdot \mathbf{b}(r \hat{\mathbf{r}}(\theta))\rangle}{\left\langle b^{2}\right\rangle}
$$

Conventionally, $\lambda$ can be viewed as an anisotropic measure of the size of the energycontaining eddies in turbulence. 
We applied the concepts summerized above to $\mathrm{H} 1$ and $\mathrm{H} 2$ data (time cadence of 40 seconds), which correspond to observations almost in the ecliptic plane, between $0.3 \mathrm{AU}$ and 1.0 AU. We analyze observations in a range from December 1974 to June 1981. We group the observations into intervals $(I)$ of 24 -hour length, and use the same procedure as in Ruiz et al. (2011) to construct an appropiate data set. For each interval, this data set contains a correlation function $\left(R^{I}\right)$ and its respective correlation length $\left(\lambda^{I}\right)$, the distance from the Sun to the spacecraft $\left(D^{I}\right)$, the SW speed and the angle $\left(\theta^{I}\right)$ between the direction of the mean magnetic field $\left\langle\mathbf{B}_{0}^{I}\right\rangle$ and the SW velocity. The number of intervals analyzed for H1 (H2) for solar maximum and solar minimum are 165 (181) and 333 (261), respectively.
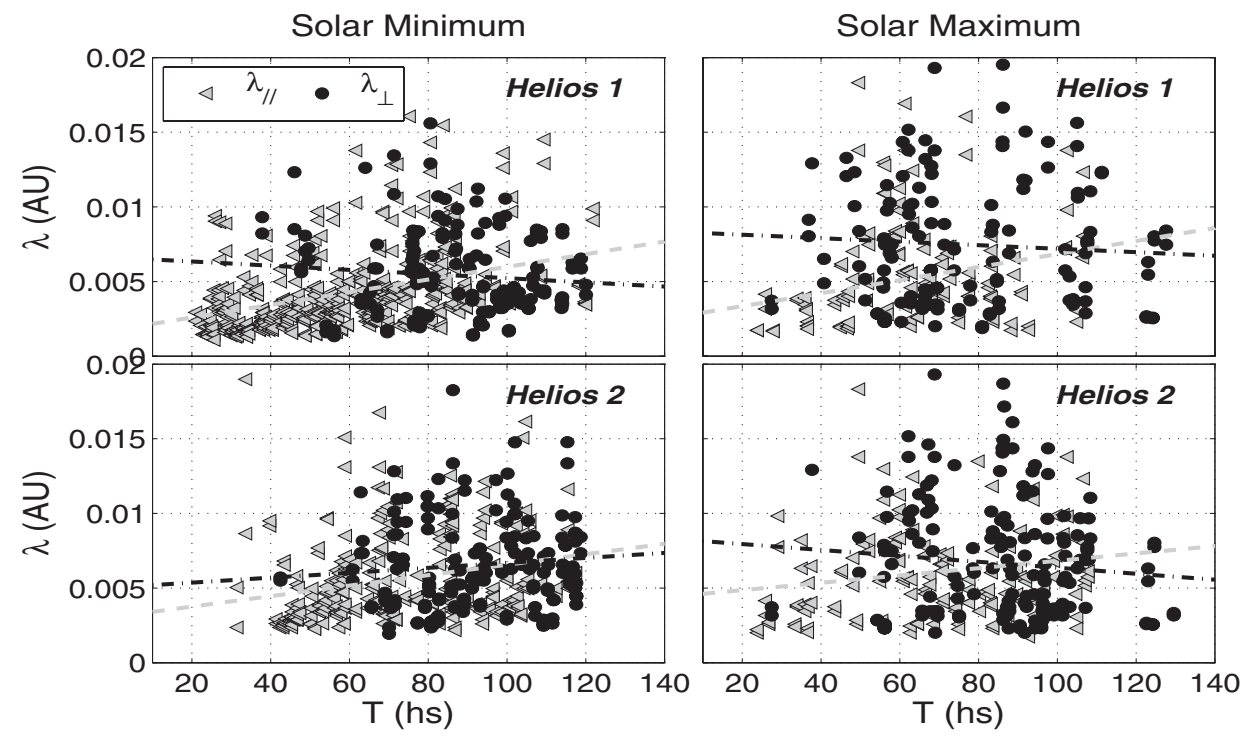

Figure 1. Scatterplot of $\lambda_{\|}$and $\lambda_{\perp}$ vs. age.

Table 1. Fitted values of the slope $\left(m\right.$, reported in $\left.10^{-5} \mathrm{AU} \mathrm{hs}^{-1}\right)$, assuming $\lambda=m T+b$.

\begin{tabular}{c|cc|cc}
\hline & \multicolumn{2}{|c|}{ Solar Minimum } & \multicolumn{2}{c}{ Solar Maximum } \\
\hline Dates & $\begin{array}{c}\text { December } \\
\text { January }\end{array}$ & 1976-December 1977 (H1) & $\begin{array}{l}\text { November 1978-June 1981 (H1) } \\
\text { December 1978-June 1981 (H2) }\end{array}$ \\
\hline & $m_{\|}$ & $m_{\perp}$ & $m_{\|}$ & $m_{\perp}$ \\
\hline Helios 1 & $4.3 \pm 1.3$ & $-1.4 \pm 1.0$ & $4.4 \pm 3.1$ & $-1.2 \pm 2.4$ \\
Helios 2 & $3.5 \pm 1.1$ & $1.7 \pm 1.1$ & $2.5 \pm 1.9$ & $-2.0 \pm 2.4$ \\
\hline
\end{tabular}

Since the degree and type of anisotropy can vary with heliodistance (Ruiz et al. 2011) and with the wind speed (Dasso et al. 2005; Weygand et al. 2011), we perform an evolution analysis of the anisotropy in correlation lengths in terms of the turbulent age of the plasma, which is simply the nominal time it takes a SW parcel moving at speed $V_{s w}^{I}$ to travel from the Sun to the spacecraft located at $D^{I}$. Then, in each interval we compute this time as $T^{I}=D^{I} / V_{s w}^{I}$, and establish two angular channels, the parallel $\left(0^{\circ}<\theta<40^{\circ}\right)$ and the perpendicular channel $\left(50^{\circ}<\theta<90^{\circ}\right)$ into which we grouped observations, thus obtaining in particular the parallel $\left(\lambda_{\|}\right)$and perpendicular $\left(\lambda_{\perp}\right)$ correlation lengths.

The relative order between $\lambda_{\|}$and $\lambda_{\perp}$ can be interpreted qualitatively in terms of the relative abundance of the two basic components of the MHD scale fluctuations, slab and 
2D. Accordingly, preponderance of the slablike component is identified by $\lambda_{\|} / \lambda_{\perp}<1$, while preponderance of the $2 \mathrm{D}$ component is identified by $\lambda_{\|} / \lambda_{\perp}>1$ (Matthaeus et al. (1990)).

Figure 1 shows the evolution of $\lambda_{\|}$and $\lambda_{\perp}$ with the turbulent age $T$, for both spacecrafts H1 (upper panels) and H2 (bottom panels), differentiating observations that correspond to solar minimum (left panels) respect to those observed in the solar maximum (right panels). Single lines, clear for $\lambda_{\|}$and dark for $\lambda_{\perp}$, are linear fits to the data. They reveal a tendency of $\lambda_{\|}$to grow with $T$. Instead, for $\lambda_{\perp}$ we find that the slope is not well defined and their values are marginally inside the error bar; it can be positive (H1 at minimum), negative (H1 at maximum) or even zero (H2 at minimum and maximum), as indicated in Table 1. Furthermore, the mean evolution of $\lambda_{\|}$and $\lambda_{\perp}$ indicates that for $T$ smaller than $\sim 70$-100 hours, the slab component of the fluctuations is dominant $\left(\lambda_{\|}<\lambda_{\perp}\right)$, while for $T$ larger than $\sim 70-100$ hours, the $2 \mathrm{D}$ component is stronger $\left(\lambda_{\|}>\lambda_{\perp}\right)$. The inversion of the relative order of both populations occurs between $T \simeq 70 \mathrm{hs}$ and $T \simeq 100 \mathrm{hs}$, and this fact is independent of the stage of the solar cycle.

The evolution of the anisotropy of correlation lengths with the aging of the solar wind $\left(T=D / V_{s w}\right)$ is consistent with the injection of alfvénic fluctuations near the Sun and a subsequent spectral transfer from modes with wavectors parallel to $\mathbf{B}_{0}\left(k_{\|}\right)$to modes with $k_{\perp}$. There is no observable dependence of this evolution with the solar cycle. During both periods of the cycle, the fluctuations evolve towards a larger abundance of the 2D-type population, with a similar age of transition between the two populations.

\section{Acknowledgements}

SD is member of the Carrera del Investigador Cientifico, CONICET. MER is a fellow of CONICET. MER and SD acknowledge support from the Argentinean grants: UBACyT 20020090100264 (UBA), PIP 11220090100825/10 (CONICET), PICT 2007-00856 (ANCPyT). SD acknowledges support from Abdus Salam International Centre for Theoretical Physics (ICTP) in the frame of his regular associateship. JW and WHM acknowledge partial support by NASA Heliophysics Guest Investigator Program grant NNX09AG31G, and NSF grants ATM-0752135 (SHINE) and ATM-0752135.

\section{References}

Belcher, J. W. \& Davis L. Jr. 1971, J. Geophys. Res., 76, 3534

Coleman, P. J. Jr. 1968, ApJ, 153, 371

Dasso, S., Milano, L. J., Matthaeus, W. H., \& Smith, C. W. 2005, ApJ 635, L181

Goldreich, P. \& S. Sridhar 1995, ApJ, 438, 763

Matthaeus, W. H., Goldstein, M. L., \& Roberts, D. A. 1990, J. Geophys. Res., 95, 20673

McComas, D. J., Elliott, H. A., Schwadron, N. A., Gosling, J. T., Skoug, R. M., \& Goldstein, B. E. $2003, G R L, 30(10), 100000-1$

Oughton, S., Priest, E. R., \& Matthaeus, W. H. (1994), J. of Fluid Mech., 280, 95

Roberts, D. Aaron, Ghosh, S., Goldstein, M. L., \& Mattheaus, W. H. (1991), Phys. Rev. Lett., 67,3741

Robinson, D. C. \& Rusbridge, M. G. 1971, Phys. of Fluids, 14, 2499

Ruiz, M. E., Dasso, S., Matthaeus, W. H., Marsch, E., \& Weygand, J. M. 2011, J. Geophys. Res. 116 (A15), 10102

Shebalin, J. V., Matthaeus, W. H., \& Montgomery, D. 1983, J. of Plasma Phys., 29, 525

Taylor, G. 1938, The Spectrum of the turbulence, in Proc. R. Soc. London Ser. A, 164, p. 476

Tu, C. Y. \& Marsch, E. 1995, MHD structures, waves and turbulence in the solar wind: observations and theories, Dordrecht: Kluwer

Weygand, J. M., Matthaeus, W. H., Dasso, S., \& Kivelson, M. G. 2011, J. Geophys. Res., 116, A08102 